\title{
Defending against Sybil Devices in Crowdsourced Mapping Services
}

\author{
Gang Wang ${ }^{\dagger}$, Bolun Wang ${ }^{\dagger}$, Tianyi Wang ${ }^{\dagger}$, Ana Nika $^{\dagger}$, Haitao Zheng ${ }^{\dagger}$, Ben Y. Zhao ${ }^{\dagger}$ \\ Department of Computer Science, UC Santa Barbara \\ ‡Department of Electronic Engineering, Tsinghua University \\ \{gangw, bolunwang, tianyi, anika, htzheng, ravenben\}@cs.ucsb.edu
}

\begin{abstract}
Real-time crowdsourced maps such as Waze provide timely updates on traffic, congestion, accidents and points of interest. In this paper, we demonstrate how lack of strong location authentication allows creation of software-based Sybil devices that expose crowdsourced map systems to a variety of security and privacy attacks. Our experiments show that a single Sybil device with limited resources can cause havoc on Waze, reporting false congestion and accidents and automatically rerouting user traffic. More importantly, we describe techniques to generate Sybil devices at scale, creating armies of virtual vehicles capable of remotely tracking precise movements for large user populations while avoiding detection. We propose a new approach to defend against Sybil devices based on co-location edges, authenticated records that attest to the one-time physical co-location of a pair of devices. Over time, colocation edges combine to form large proximity graphs that attest to physical interactions between devices, allowing scalable detection of virtual vehicles. We demonstrate the efficacy of this approach using large-scale simulations, and discuss how they can be used to dramatically reduce the impact of attacks against crowdsourced mapping services.
\end{abstract}

\section{INTRODUCTION}

Crowdsourcing is indispensable as a real-time data gathering tool for today's online services. Take for example map and navigation services. Both Google Maps and Waze use periodic GPS readings from mobile devices to infer traffic speed and congestion levels on streets and highways. Waze, the most popular crowdsourced map service, offers users more ways to actively share information on accidents, police cars, and even contribute content like editing roads, landmarks, and local fuel prices. This and the ability to interact with nearby users made Waze extremely popular, with an estimated 50 million users when it was acquired by Google for a reported \$1.3 Billion USD in June 2013. Today, Google integrates selected crowdsourced data (e.g. accidents) from Waze into its own Maps application.

Unfortunately, systems that rely on crowdsourced data are inherently vulnerable to mischievous or malicious users seeking to

Permission to make digital or hard copies of all or part of this work for personal or classroom use is granted without fee provided that copies are not made or distributed for profit or commercial advantage and that copies bear this notice and the full citation on the first page. Copyrights for components of this work owned by others than ACM must be honored. Abstracting with credit is permitted. To copy otherwise, or republish, to post on servers or to redistribute to lists, requires prior specific permission and/or a fee. Request permissions from permissions@acm.org.

MobiSys'16, June 25-30, 2016, Singapore, Singapore

(c) 2016 ACM. ISBN 978-1-4503-4269-8/16/06 . .\$15.00

DOI: http://dx.doi.org/10.1145/2906388.2906420 disrupt or game the system [41]. For example, business owners can badmouth competitors by falsifying negative reviews on Yelp or TripAdvisor, and FourSquare users can forge their physical locations for discounts $[11,54]$. For location-based services, these attacks are possible because there are no widely deployed tools to authenticate the location of mobile devices. In fact, there are few effective tools today to identify whether the origin of traffic requests are real mobile devices or software scripts.

The goal of our work is to explore the vulnerability of today's crowdsourced mobile apps against Sybil devices, software scripts that appear to application servers as "virtual mobile devices." While a single Sybil device can damage mobile apps through misbehavior, larger groups of Sybil devices can overwhelm normal users and significantly disrupt any crowdsourced mobile app. In this paper, we identify techniques that allow malicious attackers to reliably create large populations of Sybil devices using software. Using the context of the Waze crowdsourced map service, we illustrate the powerful Sybil device attack, and then develop and evaluate robust defenses against them.

While our experiments and defenses are designed with Waze (and crowdsourced maps) in mind, our results generalize to a wide range of mobile apps. With minimal modifications, our techniques can be applied to services ranging from Foursquare and Yelp to Uber and Yik Yak, allowing attackers to cheaply emulate numerous virtual devices with forged locations to overwhelm these systems via misbehavior. Misbehavior can range from falsely obtaining coupons on FourSquare/Yelp, gaming the new user coupon system in Uber, to imposing censorship on YikYak. We believe our proposed defenses can be extended to these services as well. We discuss broader implications of our work in Section 8.

Sybil attacks in Waze. In the context of Waze, our experiments reveal a number of potential attacks by Sybil devices. First is simple event forgery, where devices can generate fake events to the Waze server, including congestion, accidents or police activity that might affect user routes. Second, we describe techniques to reverse engineer mobile app APIs, thus allowing attackers to create lightweight scripts that effectively emulate a large number of virtual vehicles that collude under the control of a single attacker. We call Sybil devices in Waze "ghost riders." These Sybils can effectively magnify the efficacy of any attack, and overwhelm contributions from any legitimate users. Finally, we discover a significant privacy attack where ghost riders can silently and invisibly "follow" and precisely track individual Waze users throughout their day, precisely mapping out their movement to work, stores, hotels, gas station, and home. We experimentally confirmed the accuracy

\footnotetext{
${ }^{1}$ We refer to these scripts as Sybil devices, since they are the manifestations of Sybil attacks [16] in the context of mobile networks.
} 
of this attack against our own vehicles, quantifying the accuracy of the attack against GPS coordinates. Magnified by an army of ghost riders, an attacker can potentially track the constant whereabouts of millions of users, all without any risk of detection.

Defenses. Prior proposals to address the location authentication problem have limited appeal, because of reliance on widespread deployment of specialized hardware, either as part of physical infrastructure, i.e., cellular base stations, or as modifications to mobile devices themselves. Instead, we propose a practical solution that limits the ability of Sybil devices to amplify the potential damage incurred by any single attacker. We introduce collocation edges, authenticated records that attest to the one-time physical proximity of a pair of mobile devices. The creation of collocation edges can be triggered opportunistically by the mapping service, e.g., Waze. Over time, collocation edges combine to form large proximity graphs, network structures that attest to physical interactions between devices. Since ghost riders cannot physically interact with real devices, they cannot form direct edges with real devices, only indirectly through a small number of real devices operated by the attacker. Thus, the edges between an attacker and the rest of the network are limited by the number of real physical devices she has, regardless of how many ghost riders are under her control. This reduces the problem of detecting ghost riders to a community detection problem on the proximity graph (The graph is seeded by a small number of trusted infrastructure locations).

Our paper includes these key contributions:

- We explore limits and impacts of single device attacks on Waze, e.g., artificial congestion and events.

- We describe techniques to create light-weight ghost riders, virtual vehicles emulated by client-side scripts, through reverse engineering of the Waze app's communication protocol with the server.

- We identify a new privacy attack that allows ghost riders to virtually follow and track individual Waze users in real-time, and describe techniques to produce precise, robust location updates.

- We propose and evaluate defenses against ghost riders, using proximity graphs constructed with edges representing authenticated collocation events between pairs of devices. Since collocation can only occur between pairs of physical devices, proximity graphs limit the number of edges between real devices and ghost riders, thus isolating groups of ghost riders and making them detectable using community detection algorithms.

\section{WAZE BACKGROUND}

Waze is the most popular crowdsourced navigation app on smartphones, with more than 50 million users when it was acquired by Google in June 2013 [19]. Waze collects GPS values of users' devices to estimate real-time traffic. It also allows users to report onroad events such as accidents, road closures and police vehicles, as well as curating points of interest, editing roads, and even updating local fuel prices. Some features, e.g., user reported accidents, have been integrated into Google Maps [20]. Here, we briefly describe the key functionality in Waze as context for our work.

Trip Navigation. Waze's main feature is assist users to find the best route to their destination and turn-by-turn navigation. Waze generates aggregated real-time traffic updates using GPS data from its users, and optimizes user routes both during trip planning and during navigation. If and when traffic congestions is detected, Waze automatically re-routes users towards an alternative.
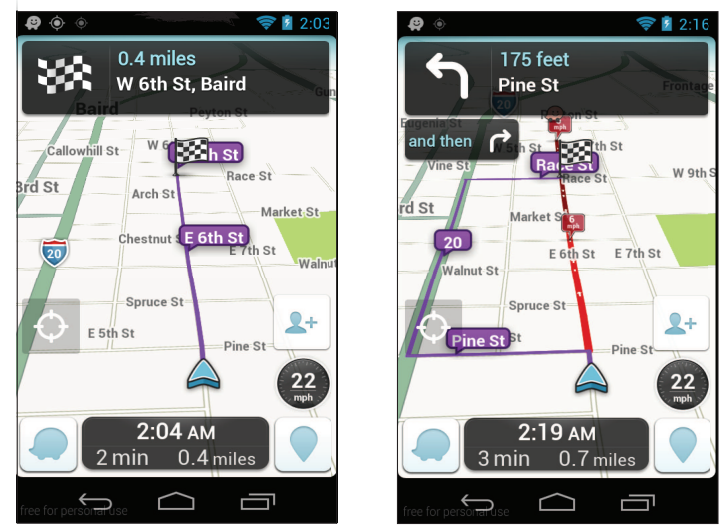

Figure 1: Before the attack (left), Waze shows the fastest route for the user. After the attack (right), the user gets automatically re-routed by the fake traffic jam.

Crowdsourced User Reports. Waze users can generate realtime event reports on their routes to inform others about ongoing incidents. Events range from accidents to road closures, hazards, and even police speed traps. Each report can include a short note with a photo. The event shows up on the map of users driving towards the reported location. As users get close, Waze pops up a window to let the user "say thanks," or report the event is "not there." If multiple users choose "not there", the event will be removed. Waze also merges multiple reports of the same event type at the same location into a single event.

Social Function. To increase user engagement, Waze supports simple social interactions. Users can see avatars and locations of nearby users. Clicking on a user's avatar shows more detailed user information, including nickname, ranking, and traveling speed. Also, users can send messages and chat with nearby users. This social function gives users the sense of a large community. Users can elevate their rankings in the community by contributing and receiving "thanks" from others.

\section{ATTACKING CROWDSOURCED MAPS}

In this section, we describe basic attacks to manipulate Waze by generating false road events and fake traffic congestion. Since Waze relies on real-time data for trip planning and route selection, these attacks can influence user's routing decisions. Attackers can attack specific users by forging congestion to force automatic rerouting on their trips. The attack is possible because Waze has no reliable authentication on user reported data, such as their device GPS.

We first discuss experimental ethics and steps we took to limit impact on real users. Then, we describe basic mechanisms and resources needed to launch attacks, and use controlled experiments on two attacks to understand their feasibility and limits. One attack creates fake road events at arbitrary locations, and the other seeks to generate artificial traffic hotspots to influence user routing.

\subsection{Ethics}

Our experiments seek to understand the feasibility and limits of practical attacks on crowdsourcing maps like Waze. We are very aware of the potential impact to real Waze users from any experiments. We consulted our local IRB and have taken all possible precautions to ensure that our experiments do not negatively impact real Waze users. In particular, we choose experiment locations 


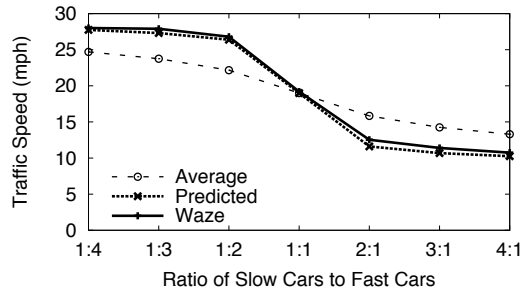

(a) Highway

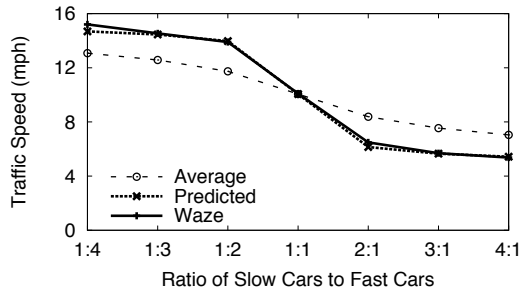

(b) Local Road

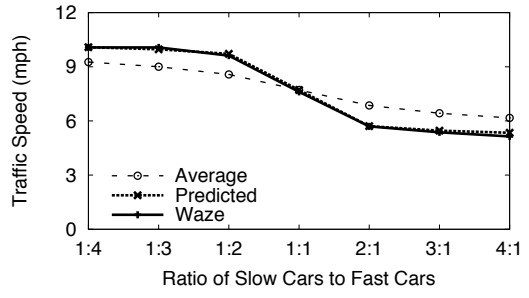

(c) Residential

Figure 2: The traffic speed of the road with respect to different combinations of number of slow cars and fast cars. We show that Waze is not using the average speed of all cars, and our inferred function can correctly predict the traffic speed displayed on Waze.

where user population density is extremely low (unoccupied roads), and only perform experiments at low-traffic hours, e.g., between $2 \mathrm{am}$ and $5 \mathrm{am}$. During the experiments, we continuously scan the entire experiment region and neighboring areas, to ensure no other Waze users (except our own accounts) are within miles of the test area. If any Waze users are detected, we immediately terminate all running experiments. Our study received the IRB approval under protocol\# COMS-ZH-YA-010-7N.

Our work is further motivated by our view of the risks of inaction versus risks posed to users by our study. On one hand, we can and have minimized risk to Waze users during our study, and we believe our experiments have not affected any Waze users. On the other hand, we believe the risk to millions of Waze users from pervasive location tracking (described in Section 5) is realistic and potentially very damaging. We feel that investigating these attacks and identifying these risks to the broad community at large was the ethically correct course of action. Furthermore, full understanding of the attacks was necessary to design an effective and practical defense. Please see Appendix A for more detailed information on our IRB approval and steps taken towards responsible disclosure.

\subsection{Basic Attack: Generating Fake Events}

Launching attacks against crowdsourced maps like Waze requires three steps: automate input to mobile devices that run the Waze app; control the device GPS and simulate device movements (e.g., car driving); obtain access to multiple devices. All three are easily achieved using widely available mobile device emulators.

Most mobile emulators run a full OS (e.g., Android, iOS) down to the kernel level, and simulate hardware features such as camera, SDCard and GPS. We choose the GenyMotion Android emulator [3] for its performance and reliability. Attackers can automatically control the GenyMotion emulator via Monkeyrunner scripts [4]. They can generate user actions such as clicking buttons and typing text, and feed pre-designed GPS sequences to the emulator (through a command line interface) to simulate location positioning and device movement. By controlling the timing of the GPS updates, they can simulate any "movement speed" of the simulated devices.

Using these tools, attackers can generate fake events (or alerts) at a given location by setting fake GPS on their virtual devices. This includes any events supported by Waze, including accidents, police, hazards, and road closures. We find that a single emulator can generate any event at arbitrary locations on the map. We validate this using experiments on a variety of unoccupied roads, including highways, local and rural roads $(50+$ locations, 3 repeated tests each). Note that our experiments only involve data in the Waze system, and do not affect real road vehicles not running the Waze app. Thus "unoccupied" means no vehicles on the road with mobile devices actively running the Waze app. After creation, the fake event stays on the map for about 30 minutes. Any Waze user can report that an event was "not there." We find it takes two consecutive "not theres" (without any "thanks" in between) to delete the event. Thus an attacker can ensure an event persists by occasionally "driving" other virtual devices to the region and "thanking" the original attacker for the event report.

\subsection{Congestion and Traffic Routing}

A more serious attack targets Waze's real-time trip routing function. Since route selection in Waze relies on predicted trip time, attackers can influence routes by creating "fake" traffic hotspots at specific locations. This can be done by configuring a group of virtual vehicles to travel slowly on a chosen road segment.

We use controlled experiments to answer two questions. First, under what conditions can attackers successfully create traffic hotspots? Second, how long can an artificial traffic hotspot last? We select three low-traffic roads in the state of Texas that are representative of three popular road types based on their speed limit-Highway (65 mph), Local (45 mph) and Residential (25 mph). To avoid real users, we choose roads in low population rural areas, and run tests at hours with the lowest traffic volumes (usually 3-5AM). We constantly scan for real users in or nearby the experimental region, and reset/terminate experiments if users come close to an area with ongoing experiments. Across all our experiments, only 2 tests were terminated due to detected presence of real users nearby. Finally, we have examined different road types and hours of the day to ensure they do not introduce bias into our results.

Creating Traffic Hotspots. Our experiment shows that it only takes one slow moving car to create a traffic congestion, when there are no real Waze users around. Waze displays a red overlay on the road to indicate traffic congestion (Figure 1, right). Different road types have different congestion thresholds, with thresholds strongly correlated to the speed limit. The congestion thresholds for Highway, Local and Residential roads are $40 \mathrm{mph}$, 20mph and $15 \mathrm{mph}$, respectively.

To understand if this is generalizable, we repeat our tests on other unoccupied roads in different states and countries. We picked 18 roads in five states in the US (CO, MO, NM, UT, MS) and British Columbia, Canada. In each region, we select three roads with different speed limits (highway, local and residential). We find consistent results: a single virtual vehicle can always generate a traffic hotspot; and the congestion thresholds were consistent across different roads of the same speed limit.

Outvoting Real Users. Generating traffic hotspot in practical scenarios faces a challenge from real Waze users who drive at normal (non-congested) speeds: attacker's virtual vehicles must "convince" the server there's a stream of slow speed traffic on the road 


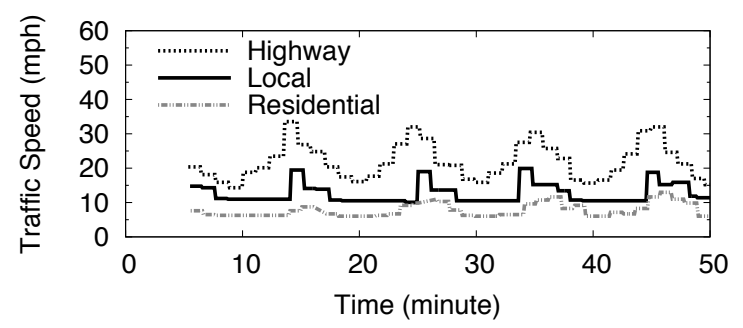

Figure 3: Long-last traffic jam created by slow cars driving-by.

even as real users tell the server otherwise. We need to understand how Waze aggregated multiple inputs to estimate traffic speed.

We perform an experiment to infer this aggregation function used by Waze. We create two groups of virtual vehicles: $N_{s}$ slowdriving cars with speed $S_{s}$, and $N_{f}$ fast-driving cars with speed $S_{f}$; and they all pass the target location at the same time. We study the congestion reported by Waze to infer the aggregation function. Note that the server-estimated traffic speed is visible on the map only if we formed a traffic hotspot. We achieve this by setting the speed tuple $\left(S_{s}, S_{f}\right)$ to $(10 \mathrm{mph}, 30 \mathrm{mph})$ for Highway, $(5,15)$ for Local and $(5,10)$ for Residential.

As shown in Figure 2, when we vary the ratio of slow cars over fast cars $\left(N_{s}: N_{f}\right)$, the Waze server produces different final traffic speeds. We observe that Waze does not simply compute an "average" speed over all the cars. Instead, it uses a weighted average with higher weight on the majority cars' speed. We infer an aggregation function as follows:

$$
S_{w a z e}=\frac{S_{\max } \cdot \max \left(N_{s}, N_{f}\right)+S_{a v g} \cdot \min \left(N_{s}, N_{f}\right)}{N_{s}+N_{f}}
$$

where $S_{\text {avg }}=\frac{S_{s} N_{s}+S_{f} N_{f}}{N_{s}+N_{f}}$, and $S_{\max }$ is the speed of the group with $N_{\max }$ cars. As shown in Figure 2, our function can predict Waze's aggregate traffic speed accurately, for all different types of roads in our test. For validation purposes, we run another set of experiments by raising $S_{f}$ above the hotspot thresholds $(65 \mathrm{mph}$, $30 \mathrm{mph}$ and $20 \mathrm{mph}$ respectively for the three roads). We can still form traffic hotspots by using more slow-driving cars $\left(N_{s}>N_{f}\right)$, and our function can still predict the traffic speed on Waze accurately.

Long-Lasting Traffic Congestion. A traffic hotspot will last for 25-30 minutes if no other cars drive by. Once aggregate speed normalizes, the congestion event is dismissed within 2-5 minutes. To create a long-lasting virtual traffic jam, attackers can simply keep sending slow-driving cars to the congestion area to resist the input from real users. We validate this using a simple, 50-minute long experiment where 3 virtual vehicles create a persistent congestion by driving slowly through an area, and then looping back every 10 minutes. Meanwhile, 2 other virtual cars emulate legitimate drivers that pass by at high speed every 10 minutes. As shown in Figure 3, the traffic hotspot persists for the entire experiment period.

Impact on End Users. Waze uses real-time traffic data to optimize routes during trip planning. Waze estimates the end-to-end trip time and recommends the fastest route. Once on the road, Waze continuously estimates the travel time, and automatically reroutes if the current route becomes congested. An attacker can launch physical attacks by placing fake traffic hotspots on the user's original route. While congestion alone does not trigger rerouting, Waze reroutes the user to a detour when the estimated travel time through the detour is shorter than the current congested route (see Figure 1).

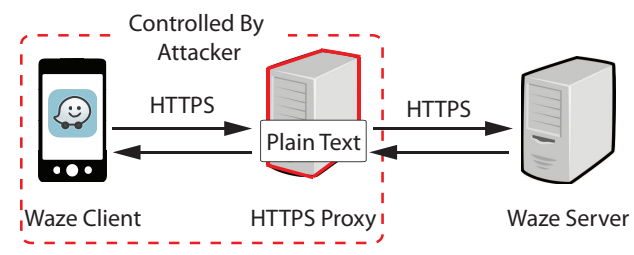

Figure 4: Using a HTTPS proxy as man-in-the-middle to intercept traffic between Waze client and server.

We also note that Waze data is used by Google Maps, and therefore can potentially impact their 1+ billion users [36]. Our experiment shows that artificial congestion do not appear on Google Maps, but fake events generated on Waze are displayed on Google Maps without verification, including "accidents", "construction" and "objects on road". Finally, event updates are synchronized on both services, with a 2-minute delay and persist for a similar period of time (e.g., 30 minutes).

\section{SYBIL ATTACKS}

So far, we have shown that attackers using emulators can create "virtual vehicles" that manipulate the Waze map. An attacker can generate much higher impact using a large group of virtual vehicles (or Sybils [16]) under control. In this section, we describe techniques to produce light-weight virtual vehicles in Waze, and explore the scalability of the group-based attacks. We refer to large groups of virtual vehicles as "ghost riders" for two reasons. First, they are easy to create en masse, and can travel in packs to outvote real users to generate more complex events, e.g., persistent traffic congestion. Second, as we show in $\$ 5$, they can make themselves invisible to nearby vehicles.

Factors Limiting Sybil Creation. We start by looking at the limits of the large-scale Sybil attacks on Waze. First, we note user accounts do not pose a challenge to attackers, since account registration can be fully automated. We found that a single-threaded Monkeyrunner script could automatically register 1000 new accounts in a day. Even though the latest version of Waze app requires SMS verification to register accounts, attackers can use older versions of APIs to create accounts without verification. Alternatively, accounts can be verified through disposable phone/SMS services [44].

The limiting factor is the scalability of vehicle emulation. Even though emulators like GenyMotion are relatively lightweight, each instance still takes significant computational resources. For example, a MacBookPro with $8 \mathrm{G}$ of RAM supports only 10 simultaneous emulator instances. For this, we explore a more scalable approach to client emulation that can increase the number of supported virtual vehicles by orders of magnitude. Specifically, we reverse engineer the communication APIs used by the app, and replace emulators with simple Python scripts that mimic API calls.

Reverse Engineering Waze APIs. The Waze app uses HTTPS to communicate with the server, so API details cannot be directly observed by capturing network traffic (TLS/SSL encrypted). However, an attacker can still intercept HTTPS traffic, by setting up a proxy [2] between her phone and Waze server as a man-in-themiddle attack [40,9]. As shown in Figure 4, an attacker needs to pre-install the proxy server's root Certificate Authorities (CA) to her own phone as a "trusted CA." This allows the proxy to present self-signed certificates to the phone claiming to be the Waze server. The Waze app on the phone will trust the proxy (since the certificate 
is signed by a "trusted CA"), and establish HTTPS connections with the proxy using proxy's public key. On the proxy side, the attacker can decrypt the traffic using proxy's private key, and then forward traffic from the phone to Waze server through a separate TLS/SSL channel. The proxy then observes traffic to the Waze servers and extracts the API calls from plain text traffic.

Hiding API calls using traffic encryption is fundamentally challenging, because the attacker has control over most of the components in the communication process, including phone, the app binary, and the proxy. A known countermeasure is certificate pinning [18], which embeds a copy of the server certificate within the app. When the app makes HTTPS requests, it validates the serverprovided certificate with its known copy before establishing connections. However, dedicated attackers can extract and replace the embedded certificate by disassembling the app binary or attaching the app to a debugger [35, 17].

Scalability of Ghost Riders. With the knowledge of Waze APIs, we build extremely lightweight Waze clients using python scripts, allocating one thread for each client. Within each thread, we $\log$ in to the app using a separate account, and maintain a live session by sending periodic GPS coordinates to the Waze server. The Python client is a full Waze client, and can report fake events using the API. Scripted emulation is highly scalable. We run 1000 virtual vehicles on a single Linux Dell PowerEdge Server (Quad Core, 2GB RAM), and find that at steady state, 1000 virtual devices only introduces a small overhead: $11 \%$ of memory usage, $2 \%$ of CPU and $420 \mathrm{Kbps}$ bandwidth. In practice, attackers can easily run tens of thousands of virtual devices on a commodity server.

Finally, we experimentally confirm the practical efficacy and scalability of ghost riders. We chose a secluded highway in rural Texas, and used 1000 virtual vehicles (hosted on a single server and single IP) to generate a highly congested traffic hotspot. We perform our experiment in the middle of the night after repeated scans showed no Waze users within miles of our test area. We positioned 1000 ghost riders one after another, and drove them slowly at $15 \mathrm{mph}$ along the highway, looping them back every 15 minutes for an entire hour. The congestion shows up on Waze 5 minutes after our test began, and stayed on the map during the entire test period. No problems were observed during our test, and tests to generate fake events (accidents etc.) also succeeded.

\section{USER TRACKING ATTACK}

Next, we describe a powerful new attack on user privacy, where virtual vehicles can track Waze users continuously without risking detection themselves. By exploiting a key social functionality in Waze, attackers can remotely follow (or stalk) any individual user in real time. This is possible with single device emulation, but greatly amplified with the help of large groups of ghost riders, possibly tracking large user populations simultaneously and putting user (location) privacy at great risk. We start by examining the feasibility (and key enablers) of this attack. We then present a simple but highly effective tracking algorithm that follows individual users in real time, which we have validated using real life experiments (with ourselves as the targets).

The only way for Waze users to avoid tracking is to go "invisible" in Waze. However, doing so forfeits the ability to generate reports or message other users. Users are also reset to "visible" each time the Waze app opens.

\subsection{Feasibility of User Tracking}

A key feature in Waze allows users to socialize with others on the road. Each user sees on her screen icons representing the loca-

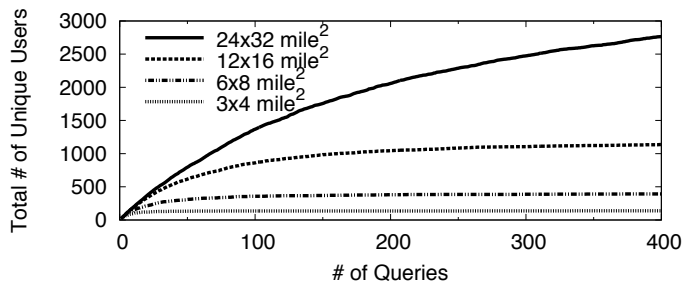

Figure 5: \# of queries vs. unique returned users in the area.

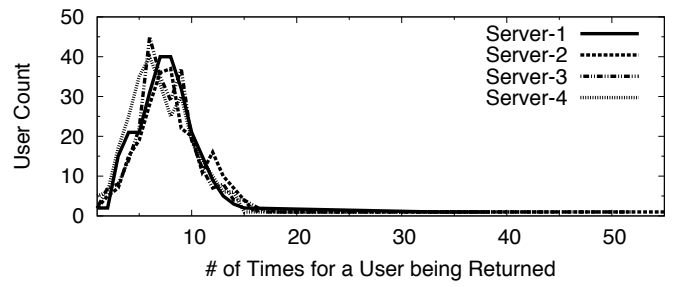

Figure 6: User's number of appearances in the returned results $\left(6 \times 8\right.$ mile $^{2}$ area $)$.

tions of nearby users, and can chat or message with them through the app. Leveraging this feature, an attacker can pinpoint any target who has the Waze app running on her phone. By constantly "refreshing" the app screen (issuing an update query to the server), an attacker can query the victim's GPS location from Waze in real time. To understand this capability, we perform detailed measurements on Waze to evaluate the efficiency and precision of user tracking.

Tracking via User Queries. A Waze client periodically requests updates in her nearby area, by issuing an update query with its GPS coordinates and a rectangular "search area." This search area can be set to any location on the map, and does not depend on the requester's own location. The server returns a list of users located in the area, including userID, nickname, account creation time, GPS coordinates and the GPS timestamp. Thus an attacker can find and "follow" a target user by first locating them at any given location (work, home) and then continuously following them by issuing update queries centered on the target vehicle location, all automated by scripts.

Overcoming Downsampling. The user query approach faces a downsampling challenge, because Waze responds to each query with an "incomplete" set of users, i.e., up to 20 users per query regardless of the search area size. This downsampled result is necessary to prevent flooding the app screen with too many user icons, but it also limits an attacker's ability to follow a moving target.

This downsampling can be overcome by simply repeatedly querying the system until the target is found. We perform query measurements on four test areas (of different sizes between $3 \times 4$ mile $^{2}$ and $24 \times 32 \mathrm{mile}^{2}$ ) in the downtown area of Los Angeles (City A, with 10 million residents as of 2015). For each area, we issue 400 queries within 10 seconds, and examine the number of unique users returned by all the queries. Results in Figure 5 show that the number of unique users reported converges after 150-250 queries for the three small search areas $\left(\leq 12 \times 16\right.$ mile $\left.^{2}\right)$. For the area of size $24 \times 32$ mile $^{2}$, more than 400 queries are required to reach convergence.

We confirm this "downsampling" is uniformly random, by comparing our measurement results to a mathematical model that projects the statistics of query results assuming uniform-random sampling. 


\begin{tabular}{|c|c|c|c|c|c|c|c|}
\hline Location & $\begin{array}{c}\text { Route } \\
\text { Length (Mile) }\end{array}$ & $\begin{array}{c}\text { Travel } \\
\text { Time (Minute) }\end{array}$ & $\begin{array}{l}\text { GPS Sent } \\
\text { By Victim }\end{array}$ & $\begin{array}{l}\text { GPS Captured } \\
\text { by Attacker }\end{array}$ & $\begin{array}{l}\text { Followed to } \\
\text { Destination? }\end{array}$ & $\begin{array}{c}\text { Avg. Track } \\
\text { Delay (Second) }\end{array}$ & $\begin{array}{l}\text { Waze User Density } \\
\left(\# \text { of Users } / \text { mile }^{2}\right)\end{array}$ \\
\hline City A & 12.8 & 35 & 18 & 16 & Yes & 43.79 & 56.6 \\
\hline Highway B & 36.6 & 40 & 20 & 19 & Yes & 9.24 & 2.8 \\
\hline
\end{tabular}

Table 1: Tracking Experiment Results.

Consider total $M$ users in the search area. The probability of a user $x$ getting sampled in a single round of query (20 users per query) is $P(x)=\frac{20}{M}$. Over $N$ queries, the number of appearances per user should follow a Binomial Distribution [25] with mean $N \cdot \frac{20}{M}$. Figure 6 plots the measured user appearances for the four servers on the $6 \times 8$ mile $^{2}$ area with $N=100$. The measured statistics follow the projected Binomial Distribution (the measured mean values closely match the theoretical expectation). This confirms that the downsampling is indeed random, and thus an attacker can recover a (near) complete set of Waze users with repeated queries. While the number of queries required increases superlinearly with area size, a complementary technique is to divide an area into smaller, fixed size partitions and query each partition's users in parallel.

We also observe that user lists returned by different Waze servers had only a partial overlap (roughly $20 \%$ of users from each server were unique to that server). This "inconsistency" across servers is caused by synchronization delay among the servers. Each user only sends its GPS coordinates to a single server which takes 2-5 minutes to propagate to other servers. Therefore, a complete user set requires queries to cover all Waze servers. At the time of our experiments, the number of Waze servers could be traced through app traffic and could be covered by a moderate number of querying accounts.

Tracking Users over Time. Our analysis found that each active Waze app updates its GPS coordinates to the server every 2 minutes, regardless of whether the user is mobile or stationary. Even when running in the background, the Waze app reports GPS values every 5 minutes. As long as the Waze app is open (even running in the background), the user's location is continuously reported to Waze and potential attackers. Clearly, a more conservative approach to managing location data would be extremely helpful here.

We note that attackers can perform long-term tracking on a target user (e.g., over months). The attacker needs a persistent ID associated to the target. The "userID" field in the metadata is insufficient, because it is a random "session" ID assigned upon user login and is released when the user kills the app. However, the "account creation time" can serve as a persistent ID, because a) it remains the same across the user's different login sessions, and b) it is precise down to the second, and is sufficiently to uniquely identify single users in the same geographic area. While Waze can remove the "account creation time" field from metadata, a persistent attacker can overcome this by analyzing the victim's mobility pattern. For example, the attacker can identify a set of locations where the victim has visited frequently or stayed during the past session, mapping to home or workplace. Then the attacker can assign a ghost rider to constantly monitor those areas, and re-identify the target once her icon shows up in a monitored location, e.g., home.

Stealth Mode. We note that attackers remain invisible to their targets, because queries on any specific geographic area can be done by Sybils operating "remotely," i.e. claiming to be in a different city, state or country. Attackers can enable their "invisible" option to hide from other nearby users. Finally, disabling these features still does not make the attacker visible. Waze only updates each user's "nearby" screen every 2 minutes (while sending its own

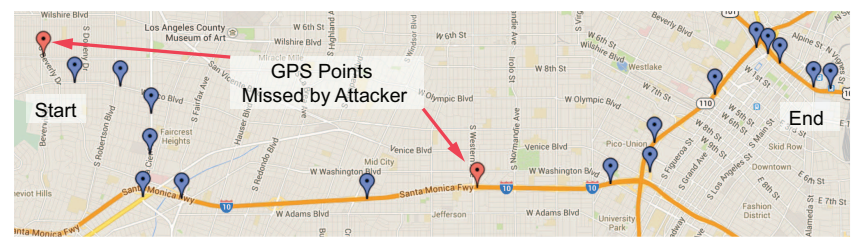

Figure 7: A graphical view of the tracking result in Los Angeles downtown (City A). Blue dots are GPS points captured by the attacker and the red dots are those missed by the attacker.

GPS update to the servers). Thus a tracker can "pop into" the target's region, query for the target, and then move out of the target's observable range, all before the target can update and detect it.

\subsection{Real-time Individual User Tracking}

To build a detailed trace of a target user's movements, an attacker first bootstraps by identifying the target's icon on the map. This can be done by identifying the target's icon while confirming her physical presence at a time and location. The attacker centers its search area on the victim's location, and issues a large number of queries (using Sybil accounts) until it captures the next GPS report from the target. If the target is moving, the attacker moves the search area along the target's direction of movement and repeats the process to get updates.

Experiments. To evaluate its effectiveness, we performed experiments by tracking one of our own Android smartphones and one of our virtual devices. Tracking was effective in both cases, but we experimented more with tracking our virtual device, since we could have it travel to any location. Using the OSRM tool [5], we generate detailed GPS traces of two driving trips, one in downtown area of Los Angeles (City A), and one along the interstate highway101 (Highway B). The target device uses a realistic driving speed based on average traffic speeds estimated by Google Maps during the experiment. The attacker used 20 virtual devices to query Waze simultaneously in a rectangular search area of size $6 \times 8$ mile $^{2}$. This should be sufficient to track the GPS update of a fast-driving car (up to $160 \mathrm{mph}$ ). Both experiments were during morning hours, and we logged both the network traffic of the target phone and query data retrieved by the attacker. Note that we did not generate any "events" or otherwise affect the Waze system in this experiment.

Results. Table 1 lists the results of tracking our virtual device, and Figure 7 presents a graphical view of the City A result. For both routes, the attacker can consistently follow the victim to her destination, though the attacker fails to capture 1-2 GPS points out of the 18-20 reported. For City A, the tracking delay, i.e., the time spent to capture the subsequent GPS of the victim, is larger (averaging 43s rather than 9s). This is because the downtown area has a higher Waze user density, and required more rounds of queries to locate the target.

Our experiments represent two highly challenging (i.e., worst case) scenarios for the attacker. The high density of Waze users 
in City A downtown is makes it challenging to locate a target in real time with downsampling. On Highway B, the target travels at a high speed $(\sim 60 \mathrm{mph})$, putting a stringent time limit on the tracking latency, i.e., the attacker must capture the target before he leaves the search area. The success of both experiments confirms the effectiveness and practicality of the proposed attack.

\section{DEFENSES}

In this section, we propose defense mechanisms to significantly limit the magnitude and impact of these attacks. While individual devices can inflict limited damage, an attacker's ability to control a large number of virtual vehicles at low cost elevates the severity of the attack in both quantity and quality. Our priority, then, is to restrict the number of ghost riders available to each attacker, thus increasing the cost per "vehicle" and reducing potential damage.

The most intuitive approach is perform strong location authentication, so that attackers must use real devices physically located at the actual locations reported. This would make ghost riders as expensive to operate as real devices. Unfortunately, existing methods for location authentication do not extend well to our context. Some proposals solely rely on trusted infrastructures (e.g., wireless access points) to verify the physical presence of devices in close proximity $[30,37]$. However, this requires large scale retrofitting of cellular celltowers or installation of new hardware, neither of which is practical at large geographic scales. Others propose to embed tamperproof location hardware on mobile devices [32, 38], which incurs high cost per user, and is only effective if enforced across all devices. For our purposes, we need a scalable approach that works with current hardware, without incurring costs on mobile users or the map service (Waze).

\subsection{Sybil Detection via Proximity Graph}

Instead of optimizing per-device location authentication, our proposed defense is a Sybil detection mechanism based on the novel concept of proximity graph. Specifically, we leverage physical proximity between real devices to create collocation edges, which act as secure attestations of shared physical presence. In a proximity graph, nodes are Waze devices (uniquely identified by an account username and password on the server side). They perform secure peer-to-peer location authentication with the Waze app running in the background. An edge is established if the proximity authentication is successful.

Because Sybil devices are scripted software, they are highly unlikely to come into physical proximity with real devices. A Sybil device can only form collocation edges with other Sybil devices (with coordination by the attacker) or the attacker's own physical devices. The resulting graph should have only very few (or no) edges between virtual devices and real users (other than the attacker). Leveraging prior work on Sybil detection in social networks, groups of Sybils can be characterized by the few "attack edges" connecting them to the rest of the graph, making them identifiable through community-detection algorithms [47].

We use a very small number of trusted nodes only to bootstrap trust in the graph. We assume a small number of infrastructure access points are known to Waze servers, e.g., hotels and public WiFi networks associated with physical locations stored in IP-location databases (used for geolocation by Apple and Google). Waze also can work with merchants that own public WiFi access points (e.g., Starbucks). These infrastructures are trusted nodes (we assume trusted nodes don't collude with attackers). Any Waze device that communicates with the Waze server under their IPs (and reports a GPS location consistent with the IP) automatically creates a new collocation edge to the trusted node.
Our Sybil defense contains two key steps. First, we build a proximity graph based on the "encounters" between Waze users (\$6.2). Second, we detect Sybils based on the trust propagation in proximity graph $(\S 6.3)$.

\subsection{Peer-based Proximity Authentication}

To build the proximity graph, we first need a reliable method to verify the physical collocation of mobile devices. We cannot rely on GPS reports since attackers can forge arbitrary GPS coordinates, or Bluetooth based device ranging [55] because the coverage is too short ( $<10$ meters) for vehicles. Instead, we consider a challengebased proximity authentication method, which leverages the limited transmission range of WiFi radios.

WiFi Tethering Challenge. We use the smartphone's WiFi radio to implement a proximity challenge between two Waze devices. Because WiFi radios have limited ranges $(<250$ meters for $802.11 \mathrm{n}$ [45])), two Waze devices must be in physical proximity to complete the challenge. Specifically, we (or the Waze server) instruct one device to enable WiFi tethering and broadcast beacons with an SSID provided by the Waze server, i.e., a randomly generated, time-varying bit string. This bit string cannot be forged by other users or used to re-identify a particular user. The second device proves its proximity to the first device by returning the SSID value heard over the air to the Waze server.

The key concerns of this approach are whether the WiFi link between two vehicles is stable/strong enough to complete the challenge, and whether the separation distance is long enough for our needs. This concern is valid given the high moving speed, potential signal blockage from vehicles' metal components, and the low transmit power of smartphones. We explore these issues with detailed measurements on real mobile devices.

First, we perform measurements on stationary vehicles to study the joint effect of blockage and limited mobile transmit power. We put two Android phones into two cars (with windows and doors closed), one running WiFi tethering to broadcast beacons and the other scanning for beacons. Figure 8 plots the WiFi beacon strength at different separation distances. We see that the above artifacts make the signal strength drop to $-100 \mathrm{dBm}$ before the distance reaches 250 meters. In the same figure, we also plot the probability of successful beacon decoding (thus challenge completion) across 400 attempts within 2 minutes. It remains $100 \%$ when the two cars are separated by $<80$ meters, and drops to zero at 160 meters.

Next, we perform driving experiments on a highway at normal traffic hours in the presence of other vehicles. The vehicles travel at speeds averaging $65 \mathrm{mph}$. During driving, we are able to vary the distance between the two cars, and use recorded GPS logs to calculate the separation distance. Figure 9 shows that while WiFi signal strength fluctuates during our experiments, the probability of beacon decoding remains very high at $98 \%$ when the separation is less than 80 meters but drops to $<10 \%$ once the two cars are more than 140 meters apart.

Overall, the results suggest the proposed WiFi tethering challenge is a reliable method for proximity authentication for our system. In practice, Waze can start the challenge when detecting the two vehicles are within the effective range, e.g., 80 meters. Since the WiFi channel scan is fast, e.g., 1-2 seconds to do a full channel scan in our experiments, this challenge can be accomplished quickly with minimum energy cost on mobile devices. It is easy to implement this scheme using existing APIs to control WiFi radio to open tethering (setWifiApEnabled API in Android).

Constructing Proximity Graphs. In a proximity graph, each node is a Waze device, and an edge indicates the two users come 


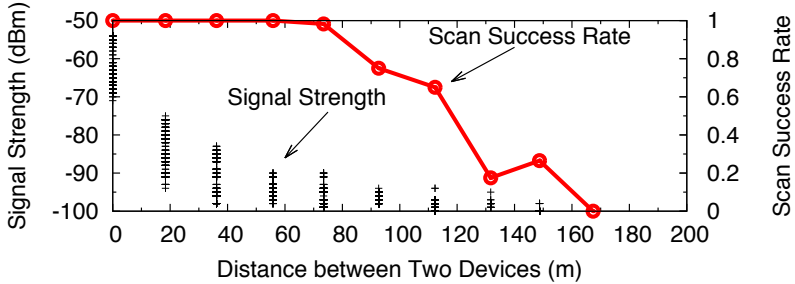

Figure 8: WiFi signal strength and scan success rate with respect to car distance in static scenarios.

into physical proximity, e.g., 80 meters, within a predefined time window. The resulting graph is undirected but weighted based on the number of times the two users have encountered. Using weighted graph makes it harder for Sybils to blend into the normal user region. Intuitively, real users will get more weights on their edges as they use Waze over time. For attackers, in order to blend in the graph, they need to build more weighted attack edges to real users (higher costs).

This approach should not introduce much energy consumption to users' phones. First, Waze server does not need to trigger collocation authentication every time two users are in close proximity. Instead, the proximity graph will be built up over time. A user only need to authenticate with other users occasionally, since we can require that device authentication expires after a moderate time period (e.g., months) to reduce the net impact on wireless performance and energy usage. Second, since the process is triggered by the Waze server, Waze can can use WiFi sensing from devices to find "opportunistic" authentication times that minimize impact on performance and energy. Waze can also use one tether to simultaneously authenticate multiple colocated devices within an area. This further reduces authentication overhead, and avoids performance issues like wireless interference in areas with high user density.

\subsection{Graph-based Sybil Detection}

We apply graph-based Sybil detection algorithms to detect Sybils in Waze proximity graph. Graph-based Sybil detectors [53, 52, 47, $14,10]$ were originally proposed in social networks. They all rely on the key assumption that Sybils have difficulty to form edges with real users, which results in a sparse cut between the Sybil and nonSybil regions in the social graph. Because of the limited number of "attack edges" between Sybils and non-Sybils, a random walk from non-Sybil region has a higher landing probability to land on a nonSybil node than a Sybil node. Our proximity graph holds the same assumption that these algorithms require - with the WiFi proximity authentication, it's difficult for Sybil devices (ghost riders) to build attack edges to real Waze users.

SybilRank. Among available algorithms, we use SybilRank [10]. Compared to its counterparts (SybilGuard [53], SybilLimit [52] and SybilInfer [14]), SybilRank achieves higher accuracy at a lower computational cost. At the high-level, its counterparts need to perform actual random walks, which is very costly and yet often gives incomplete views of the graph. Instead, SybilRank uses power iteration [28] to compute the random walk landing probability for all nodes. This significantly boosts the algorithm accuracy and speed. Furthermore, SybilRank has a better tolerance on community structures in the non-Sybil region (for using multiple trusted nodes), making it more suitable for real-world graphs.

As context, we briefly describe how SybilRank works and refer readers to [10] for more details. SybilRank ranks the nodes based

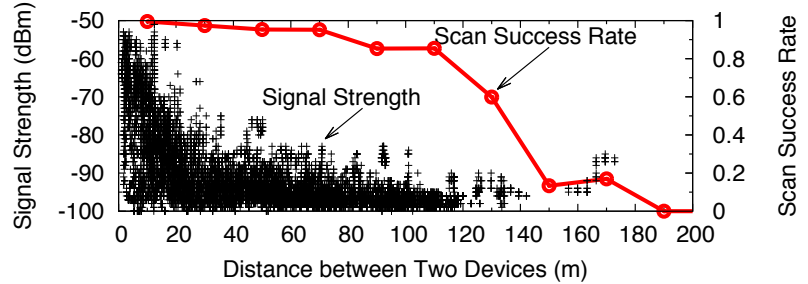

Figure 9: WiFi signal strength and scan success rate with respect to car distance in driving scenarios.

on how likely they are Sybils. The algorithm starts with multiple trusted nodes in the graph. It iteratively computes the landing probability for short random walks (originated from trusted nodes) to land on all other nodes. The landing probability is normalized by the node's degree, which acts as the trust score for ranking. Intuitively, short random walks from trusted nodes are very unlikely to traverse the few attack edges to reach Sybil nodes, thus the ranking scores of Sybils should be lower. For Sybil detection, Waze can set a cutoff threshold on the trust score, and label the tail of the ranked list as Sybils.

The original SybilRank works on unweighted social graphs. We modified it to work on our weighted proximity graph: when a node propagates trust (or performs random walks) to its neighbors, instead of splitting the trust equally, it distributes proportionally based on the edge weights. This actually makes it harder for Sybils to evade SybilRank - they will need to build more high-weight attack edges to real users to receive trust.

\section{COUNTERMEASURE EVALUATION}

We use simulations to evaluate the effectiveness of our proposed defense. We focus on evaluating the feasibility and cost for attackers to maintain a large number of Sybils after the Sybil detection is in place. We quantify the cost by the number of attack edges a Sybil must establish with real users. In practice, this translates into the effort taken to physically drive around and use physical devices (with WiFi radios) per Sybil to complete proximity authentication. In the following, we first describe our simulation setup, and then present the key findings and their implications on Waze.

\subsection{Evaluation Setup}

We first discuss how we construct a synthetic proximity graph for our evaluation, followed by the counter strategies taken by attackers to evade detection. Finally, we describe the evaluation metrics for Sybil detection.

Simulating Proximity Graphs. We use well-known models on human encountering to create synthetic proximity graphs. This is because, to the best of our knowledge, there is no public per-user mobility dataset with sufficient scale and temporal coverage to support our evaluation. Also, directly crawling large-scale, per-user mobility trace from Waze can lead to questionable privacy implications, and thus we exclude this option.

Existing literatures [33, 13, 43, 23, 29] all suggest that human (and vehicle) encounter patterns display strong scale-free and "smallworld" properties [6]. Thus we follow the methodology of [33] to simulate a power-law based encounter process among Waze users. Given a user population $N$, we first assign each user an encounter probability following a power-law distribution ( $\alpha=2$ based on the empirical values $[33,12])$. We then simulate user encounter over 


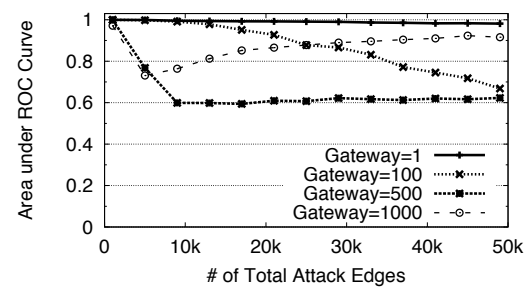

(a) Sybil inner connection avg. degree $=5$

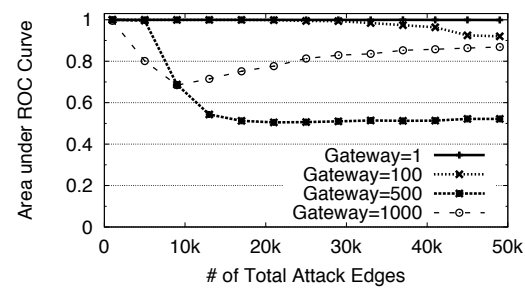

(b) Sybil inner connection avg. degree $=10$

Figure 10: AUC with respect to number of attack edges, where Sybils form power-law inner connections.

time, by adding edges to the graph based on the joint probability of the two nodes.

For our evaluation, we produce a proximity graph for $N=$ 10000 normal users and use the snapshot when $99.9 \%$ of nodes are connected. Note that as the graph gets denser over time, it is harder for Sybils to blend into normal user regions. We use this graph to simulate the lower-bound performance of Sybil detection. ${ }^{2}$ As a potential limitation, the simulated graph parameters might be different for different cities of Waze. Thus we don't claim our reported numbers will exactly match what Waze produces. The idea is that Waze can follow our methodology to run the same experiments on their real graphs.

Attacker Models. In the presence of Sybil detection, an attacker will try mixing their Sybils into the proximity graph. We consider the following strategies:

1. Single-Gateway - An attacker first takes one Sybil account (as the gateway) to build attack edges to normal users. Then the attacker connects the remaining Sybils to this gateway. In practice, this means the attacker only needs to take one physical phone to go out and encounter normal users.

2. Multi-Gateways - An attacker distributes the attack edges to multiple gateways, and then evenly spreads the other Sybils across the gateways. This helps the Sybils to blend in with normal users. The attacker pays an extra cost in terms of using multiple real devices to build attack edges.

The attacker also builds edges among its own Sybils. This incurs no additional cost since Sybils can easily collude to pass proximity authentication, but introduces key benefits. First, it makes Sybils' degree distribution appear more legitimate. Second, it can potentially increase Sybils' trust score: when a random walk reaches one Sybil node, its edges to the fellow Sybils help to sustain the random walk within the Sybil region. In our simulation, we follow the scale-free distribution to add edges among Sybils mimicking normal user region (we did not use a fully connected network between Sybils since it is more easily detectable).

Evaluation Metrics. To evaluate Sybil detection efficacy, we use the standard false positive (negative) rate, and the Area under the Receiver Operating Characteristic curve (AUC) used by SybilRank [10]. AUC represents the probability that SybilRank ranks a random Sybil node lower than a random non-Sybil node. Its value ranges from 0 to 1, where 1 means the ranking is perfect (all Sybils are ranked lower than non-Sybils), 0 means the ranking is always flipped, and 0.5 matches the result of random guessing. Compared to false positive (negative) rates, AUC is independent of the cutoff threshold, and thus comparable across experiment settings.

\footnotetext{
${ }^{2}$ Validated by experiments: a denser, $99.99 \%$ connected graph can uniformly improve Sybil detection accuracy.
}

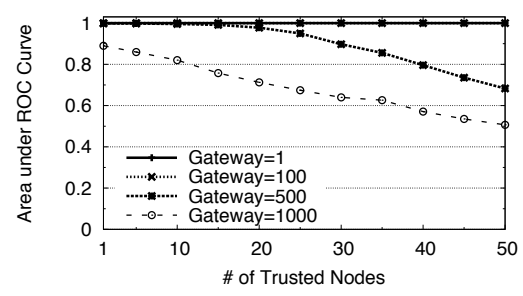

Figure 11: Impact of \# of trusted nodes (average degree $=\mathbf{1 0}$ for Sybil region; $5 \mathrm{~K}$ attack edges).

\subsection{Results}

Accuracy of Sybil Detection. We assume the attacker seeks to embed 1000 Sybils into the proximity graph. We use either singleor multi-gateway approaches to build attack edges on the proximity graph by connecting Sybils to randomly chosen normal users. We then add edges between Sybil nodes, following the power-law distribution and producing an average weighted degree of either 5 or 10 (to emulate different Sybil subgraph density). We randomly select 10 trusted nodes to bootstrap trust for SybilRank and run it on the proximity graph. We repeat each experiment 50 times.

Figure 10 shows that the Sybil detection mechanism is highly effective. For attackers of the single-gateway model, the AUC is very close to 1 ( $>0.983)$, indicating Waze can identify almost all Sybils even after the attacker established a large number of attack edges, e.g., 50000. Meanwhile, the multi-gateway method helps attackers add "undetected" Sybils, but the number of gateways required is significant. For example, to maintain 1000 Sybils, i.e., by bringing down AUC to 0.5, the attacker needs at least 500 as gateways. In practice, this means wardriving with $500+$ physical devices to meet real users, which is a significant overhead.

Interestingly, the 1000-gateway result (where every Sybil is a gateway) shows that, at certain point, adding more attack edges can actually hurt Sybils. This is potentially due to the fact that SybilRank uses node degree to normalize trust score. For gateways that connect to both normal users and other Sybils, the additional "trust" received by adding more attack edges cannot compensate the penalty of degree normalization.

For a better look at the detection accuracy, we convert the AUC in Figure 10(b) to false positives (classifying real users as Sybils) and false negatives (classifying Sybils as real users). For simplicity, we set a cutoff value to mark the bottom $10 \%$ of the ranked nodes as Sybils. ${ }^{3}$ As shown in Figure 12, SybilRank is highly accurate to detect Sybils when the number of gateways is less than 100. Again 100 gateways incurs high cost in practice.

Next we quickly examine the impact of trusted nodes to Sybil detection. Figure 11 shows a small number of trusted node is enough to run SybilRank. Interestingly, adding more trusted nodes can slightly hurt Sybil detection, possibly because it gives the attacker (gateways) a higher chance to receive trust. In practice, multiple trusted nodes can help SybilRank overcome potential community structures in proximity graph (e.g., users of the same city form a cluster). So Waze should place trusted nodes accordingly to cover geographic clusters.

Cost of Sybil Attacks. Next, we infer the rough cost of attackers on implementing successful Sybil attacks. For this we look at

\footnotetext{
${ }^{3}$ This cutoff value is only to convert the error rate. In practice, Waze can optimize this value based on the trust score or manual examination.
} 


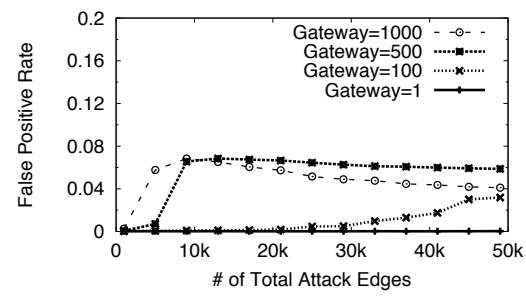

(a) False Positive Rate

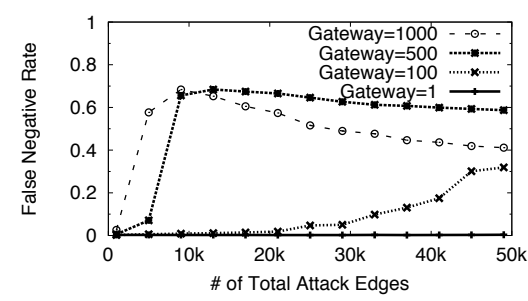

(b) False Negative Rate

Figure 12: Detection error rates with respect to number of attack edges. We set average degree $=10$ for Sybils' power-law inner connections.

the number of attack edges required to successfully embed a given number of Sybils. Our experiment assumes the attacker uses 500 gateways and builds power-law distributed inner connections with average degree $=10$. Figure 13 shows the number of attack edges required to achieve a specific AUC under SybilRank as a function of the target number of Sybils. We see that the attack edge count increases linearly with the Sybil count. The cost of Sybil attack is high: to maintain 3000 Sybils, the attacker must make 60,000 attack edges to keep AUC below 0.75, and spread these attack edges across 500 high-cost gateways.

Smaller Sybil Groups. Finally, we examine how effective our system is in detecting much smaller Sybil groups. We test Sybil groups with size of 20,50 and 100 using a single-gateway approach. We configure $50 \mathrm{~K}$ attacking edges for Sybils with inner degree $=10$. The resulting AUC of Sybil detection is $0.90,0.95$ and 0.99 respectively. This confirms our system can effectively identify small Sybil groups as well.

\subsection{Implications on Waze}

These results show that our Sybil detection method is highly effective. It significantly increases the cost (in purchasing physical devices and time spent actually driving on the road) to launch Sybil attacks. Also, SybilRank is scalable enough for large systems like Waze. A social network with tens of millions of users has been running SybilRank on Hadoop servers [10].

In addition to Sybil detection, Waze can incorporate other mechanisms to protect its users. We briefly describe a few key ideas, but leave the integration with our approach to future work. First, IP verification: when a user claims she is driving, Waze can examine whether her IP is a mobile IP that belongs to a valid cellular carrier or a suspicious web proxy. However, this approach is ineffective if dedicated attackers route the attack traffic through a cellular data plan. Second, strict rate limit: with that, attackers will need to run more Sybil devices to implement the same attack. Third, verifications on account registration: this needs to be handled carefully since email/SMS based verification can be bypassed using disposable email or phone numbers [44]. Finally, detecting extremely inconsistent GPS/event reports. The challenge, however, is to distinguish honest reports from the fake ones since attacker can easily outvote real users. If Waze chooses to ignore all the inconsistent reports, it will lead to DOS attack where attackers disable the service with inconsistent data.

\section{BROADER IMPLICATIONS}

While our experiments and defenses have focused strictly on Waze, our results are applicable to a wider range of mobile applications that rely on geolocation for user-contributed content and metadata. Examples include location based check-in and review services (Foursquare, Yelp), crowdsourced navigation systems (Waze,

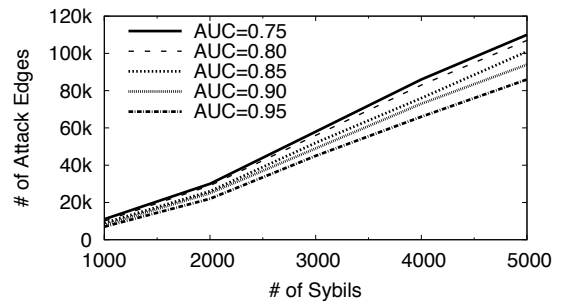

Figure 13: \# of attack edges needed to maintain $x$ Sybil devices with respect to different AUC level.
Moovit), crowdsourced taxi services (Uber, Lyft), mobile dating apps (Tinder, Bumble) and anonymous mobile communities (Yik Yak, Whisper).

These systems face two common challenges exposing them to potential attacks. First, our efforts show that it is difficult for app developers to build a truly secure channel between the app and the server. There are numerous avenues for an attacker to reverseengineer and mimic an app's API calls, thereby creating "cheap" virtual devices and launching Sybil attack [16]. Second, there are no deployed mechanisms to authenticate location data (e.g., GPS report). Without a secure channel to the server and authenticated location, these mobile apps are vulnerable to automated attacks ranging from nuisance (prank calls to Uber) to malicious content attacks (large-scale rating manipulation on Yelp).

To validate our point, we run a quick empirical analysis on a broad class of mobile apps to understand how easy it is to reverseengineer their APIs and inject falsified data into the system. We pick one app from each category including Foursquare, Uber, Tinder and Yik Yak (an incomplete list). We find that, although all the listed apps use TLS/SSL to encrypt their network traffic, their APIs can be fully exposed by the method in $\S 4$. For each app, we were able to build a light-weight client using python script, and feed arbitrary GPS to their key function calls. For example, with forged GPS, a group of Foursquare clients can deliver large volumes of check-ins to a given venue without physically visiting it; On Uber, one can distribute many virtual devices as sensors, and passively monitor and track all drivers (and their passengers) within a large area (see §5). Similarly for Yik Yak and Tinder, the virtual devices make it possible to perform wardriving in a given location area to post and collect anonymous Yik Yak messages or Tinder profiles. In addition, apps like Tinder also display the geographical distance to a nearby user (e.g., 1 mile). Attacker can use multiple virtual devices to measure the distance to the target user, and "triangulate" that user's exact location [50]. There are possible app-specific defenses, and we leave their design and evaluation to future work.

\section{DISCLOSURE AND IMPACT}

Before the first writeup of our work, we sought to inform the Google Waze team of our results. We first used multiple existing Google contacts on the security and Android teams. When that failed to reach the Waze team, we got in touch with Niels Provos, who then relayed information about our project to the Waze team.

Through our periodic tests of the Waze app, we noticed recent updates made significant changes to how the app reports user location data to the server (and other users). In the new Waze update (v4.4.0, released in April 2016), the app only reports user GPS values when the user is actively driving (moving at a moderate/fast rate of speed). GPS tracking stops when a user is walking or standing still. In addition, Waze automatically shuts down if the user puts it 
in the background, and has not driven for a while. To resume user tracking (GPS reporting), users must manually bring the app to the foreground. Finally, Waze now hide users' starting and destination locations of their trips.

While online documentation claims that these optimizations are to reduce energy usage for the Waze app, we are gratified by the dramatic steps taken to limit user tracking and improve user privacy. These changes dramatically reduce the amount of GPS data sent to the server (and made available to potential attackers through the API). By our estimates, the update reduces the amount of GPS tracking data for a typical user by nearly a factor of $10 x$. In addition, removing the first and last GPS values of a trip means that it is significantly harder to track a user through multiple trips. Previously, users could be tracked across new Waze sessions, despite new per-session identifiers, by matching the destination point of one trip with the starting point of the next.

We note that while Waze has taken significantly steps to improve user privacy, users can still be tracked while they are actively using the app. More importantly, the attack we identified here can still wreak havoc with a wide range of mobile apps, and Sybil devices are a real challenge still in need of practical solutions. We hope our work spurs future work to address this problem.

\section{RELATED WORK}

Security in Location-based Services. Location-based services face various threats, ranging from rogue users reporting fake GPS [11,22], to malicious parties compromising user privacy [15, 26, 27]. A related study on Waze [39] demonstrated that smallscale attacks can create traffic jams or track user icons, with up to 15 mobile emulators. Our work differs in two key aspects. First, we show that it's possible to reverse engineer its APIs, enabling lightweight Sybil devices (simple scripts) to replace full-stack emulators. This increase the scale of potential attacks by orders of magnitude, to thousands of Waze clients per commodity laptop. The impact of thousands of virtual vehicles is qualitatively different from 10-15 mobile simulators. Second, as possible defenses, [39] cites known tools such as phone number/IP verification, or location authentication with cellular towers, which have limited applicability (see §6). In contrast, we propose a novel proximity graph approach to detect and constrain the impact of virtual devices.

Researchers have proposed to preserve user location privacy against map services such as Waze and Google. Earlier studies apply location cloaking by adding noise to the GPS reports [21]. Recent work use zero-knowledge [24] and differential privacy [8] to preserve the location privacy of individual users, while maintaining user accountability and the accuracy of aggregated statistics. Our work differs by focusing on the attacks against the map services.

Mobile Location Authentication. Defending against forged GPS is challenging. One direction is to authenticate user locations using wireless infrastructures: WiFi APs [30,37], cellular base stations [30,37] and femtocells [7]. Devices must come into physical proximity to these infrastructures to be authenticated. But it requires cooperation among a wide range of infrastructures (also modifications to their software/hardware), which is impractical for large-scale services like Waze. Our work only uses a small number of trusted infrastructures to bootstrap, and relies on peer-based trust propagation to achieve coverage. Other researchers have proposed "peer-based" methods to authenticate collocated mobile devices [42, 51, 55, 31, 34]. Different from existing work, we use peer-based collocation authentication to build proximity graphs for Sybil detection, instead of directly authenticating a device's physical location.
Sybil Detection. Sybil detection has been studied in P2P networks [16] and online social networks [47, 49, 48]. The most popular approach is graph-based where the key assumption is that Sybils have difficulty to connect to real users [10, 14, 46, 52, 53]. Thus Sybils would form a well-connected subgraph that has a small quotient-cut from the non-Sybil region. Our work constructs a proximity graph that holds the same assumption, and applies Sybil detection algorithm to locate ghost riders in Waze. We differ from [10] in the graph used and the attack models.

\section{CONCLUSION}

We describe our efforts to identify and study a range of attacks on crowdsourced map services. We identify a range of single and multi-user attacks, and describe techniques to build and control groups of virtual vehicles (ghost riders) to amplify these attacks. Our work shows that today's mapping services are highly vulnerable to software agents controlled by malicious users, and both the stability of these services and the privacy of millions of users are at stake. While our study and experiments focus on the Waze system, we believe the large majority of our results can be generalized to crowdsourced apps as a group. We propose and validate a suite of techniques that help services build proximity graphs and use them to effectively detect Sybil devices.

Throughout this work, we have taken active steps to isolate our experiments and prevent any negative consequence on real Waze users. We also used our existing Google/Waze contacts to inform Waze team of our results. More details on IRB, ethics and disclosure are contained in Appendix A.

\section{Acknowledgments}

We would like to thank our shepherd Z. Morley Mao and the anonymous reviewers for their comments. This project was supported by NSF grants CNS-1527939 and CNS-1224100. Any opinions, findings, and conclusions or recommendations expressed in this material are those of the authors and do not necessarily reflect the views of any funding agencies.

\section{Appendix A - IRB, Ethics}

Our study was reviewed and approved by our local IRB. Prior to doing any real measurements on the system, we submitted a human subject protocol for approval by our institutional IRB. The protocol was fully reviewed for ethics and privacy risks, and the response was our study can be exempt. We put the request into our IRB system and began our work. Then the confirmation of exemption arrived and our study received the IRB approval under protocol \# COMS-ZH-YA-010-7N.

As described in the paper, we are very aware of the potential impact on real Waze users from any experiments. We took very careful precautions to ensure that our experiments will not negatively impact Waze servers or Waze users. In particular, we conducted numerous measurements of diverse traffic regions (read-only) to locate areas of extremely low traffic density. We chose experiment locations where user population density is extremely low (unoccupied roads), and only perform experiments at low-traffic hours, e.g. between 3 am and 5am. During experiments, we continuously scan the entire region including our experimental area and neighboring regions, to ensure no other Waze users (except our own accounts) are within miles of the test area. If any Waze users are detected, we immediately terminate any running experiments. We took care to limit congestion tests to areas with lots of local route redundancy, thus we would not affect the routing of any long distance 
trips (e.g. taking highway 80 because the 101 was congested). Finally, while we cannot detect invisible users in our test area, we have taken every precaution to only test on roads and times that show very little traffic, e.g. low population areas at 4 am local time. We believe in practice, invisible users make up a small subset of the Waze population, because they cannot send reports or message other users (effectively removing most/all of the social functionality in Waze), and Waze resets the invisible setting every time the app is opened [1].

\section{REFERENCES}

[1] About Waze: Privacy. https://support.google.com/waze/answer/6071193?hl=en.

[2] Charles Proxy. http://www.charlesproxy.com.

[3] GenyMotion Emulator. http://www.genymotion.com.

[4] Monkeyrunner. http://developer.android.com/tools/ help/monkeyrunner_concepts.html.

[5] Open Source Routing Machine (OSRM). http://map.project-osrm.org.

[6] A.-L. Barabasi and R. Albert. Emergence of scaling in random networks. Science, 286, 1999.

[7] J. Brassil, P. K. Manadhata, and R. Netravali. Traffic signature-based mobile device location authentication. IEEE Transactions on Mobile Computing, 13(9):2156-2169, 2014.

[8] J. W. S. Brown, O. Ohrimenko, and R. Tamassia. Haze: Privacy-preserving real-time traffic statistics. In Proc. of SIGSPATIAL, 2013.

[9] C. Brubaker, S. Jana, B. Ray, S. Khurshid, and V. Shmatikov. Using frankencerts for automated adversarial testing of certificate validation in ssl/tls implementations. In Proc. of IEEE S\&P, 2014.

[10] Q. Cao, M. Sirivianos, X. Yang, and T. Pregueiro. Aiding the detection of fake accounts in large scale social online services. In Proc. of NSDI, 2012.

[11] B. Carbunar and R. Potharaju. You unlocked the mt. everest badge on foursquare! countering location fraud in geosocial networks. In Proc. of MASS, 2012.

[12] A. Clauset, C. R. Shalizi, and M. E. Newman. Power-law distributions in empirical data. SIAM review, 51(4):661-703, 2009.

[13] F. Cunha, A. C. Viana, R. A. F. Mini, and A. A. F. Loureiro. Is it possible to find social properties in vehicular networks? In Proc. of ISCC, 2014.

[14] G. Danezis and P. Mittal. Sybilinfer: Detecting sybil nodes using social networks. In Proc of NDSS, 2009.

[15] Y.-A. de Montjoye, M. Verleysen, and V. D. Blondel. Unique in the crowd: The privacy bounds of human mobility. Scientific Reports, 3, 2013.

[16] J. R. Douceur. The Sybil attack. In Proc. of IPTPS, 2002.

[17] W. Enck, D. Octeau, P. McDaniel, and S. Chaudhuri. A study of android application security. In Proc. of USENIX Security, 2011.

[18] S. Fahl, M. Harbach, H. Perl, M. Koetter, and M. Smith. Rethinking ssl development in an appified world. In Proc. of CCS, 2013.

[19] V. Goel. Maps that live and breathe with data. The New York Times, June 2013.

[20] Google. Google maps and waze, outsmarting traffic together. Google Official Blog, June 2013.

[21] M. Gruteser and D. Grunwald. Anonymous usage of location-based services through spatial and temporal cloaking. In Proc. of MobiSys, 2003.
[22] W. He, X. Liu, and M. Ren. Location cheating: A security challenge to location-based social network services. In Proc. of ICDCS, 2011.

[23] T. Hossmann, T. Spyropoulos, and F. Legendre. Know thy neighbor: Towards optimal mapping of contacts to social graphs for dtn routing. In Proc. of INFOCOM, 2010.

[24] T. Jeske. Floating car data from smartphones: What google and waze know about you and how hackers can control traffic. Black Hat, 2013.

[25] V. Kachitvichyanukul and B. W. Schmeiser. Binomial random variate generation. Commun. ACM, 31(2):216-222, 1988.

[26] J. Krumm. Inference attacks on location tracks. In Pervasive Computing. 2007.

[27] J. Krumm. A survey of computational location privacy. Personal and Ubiquitous Computing, 2009.

[28] A. Langville and C. Meyer. Deeper inside pagerank. Internet Mathematics, 1(3):335-380, 2004.

[29] X. Liu, Z. Li, W. Li, S. Lu, X. Wang, and D. Chen. Exploring social properties in vehicular ad hoc networks. In Proc. of Internetware, 2012.

[30] W. Luo and U. Hengartner. Proving your location without giving up your privacy. In Proc. of HotMobile, 2010.

[31] J. Manweiler, R. Scudellari, and L. P. Cox. Smile: Encounter-based trust for mobile social services. In Proc. of CCS, 2009.

[32] C. Marforio, N. Karapanos, C. Soriente, and K. Capkun. Smartphones as practical and secure location verification tokens for payments. In Proc. of NDSS, 2014.

[33] A. G. Miklas, K. K. Gollu, K. K. W. Chan, S. Saroiu, K. P. Gummadi, and E. de Lara. Exploiting social interactions in mobile systems. In Proc. of Ubicomp, 2007.

[34] A. Narayanan, N. Thiagarajan, M. Lakhani, M. Hamburg, and D. Boneh. Location Privacy via Private Proximity Testing. In Proc. of NDSS, 2011.

[35] J. Osborne and A. Diquet. When security gets in the way: Pentesting mobile apps that use certificate pinning. Black Hat, 2012.

[36] B. Reed. Google maps becomes google's second 1 billion-download hit. Yahoo! News, June 2014.

[37] S. Saroiu and A. Wolman. Enabling new mobile applications with location proofs. In Proc. of HotMobile, 2009.

[38] S. Saroiu and A. Wolman. I am a sensor, and i approve this message. In Proc. of HotMobile, 2010.

[39] M. B. Sinai, N. Partush, S. Yadid, and E. Yahav. Exploiting social navigation. Black Hat Asia, CoRR:abs/1410.0151, 2015.

[40] D. Sounthiraraj, J. Sahs, G. Greenwood, Z. Lin, and L. Khan. Smv-hunter: Large scale, automated detection of ssl/tls man-in-the-middle vulnerabilities in android apps. In Proc. of NDSS, 2014.

[41] N. Stefanovitch, A. Alshamsi, M. Cebrian, and I. Rahwan. Error and attack tolerance of collective problem solving: The darpa shredder challenge. EPJ Data Science, 3(1):1-27, 2014.

[42] M. Talasila, R. Curtmola, and C. Borcea. LINK: location verification through immediate neighbors knowledge. In Proc. of MobiQuitous, 2010.

[43] F. Tan, Y. Borghol, and S. Ardon. Emo: A statistical encounter-based mobility model for simulating delay tolerant networks. In Proc. of WOWMOM, 2008. 
[44] K. Thomas, D. Iatskiv, E. Bursztein, T. Pietraszek, C. Grier, and D. McCoy. Dialing back abuse on phone verified accounts. In Proc. of CCS, 2014.

[45] J. M. Tjensvold. Comparison of the IEEE 802.11, 802.15.1,802.15.4 and 802.15.6 wireless standards. http://janmagnet.files.wordpress.com/2008/07/ comparison-ieee-802-standards.pdf, 2007.

[46] N. Tran, B. Min, J. Li, and L. Subramanian. Sybil-resilient online content voting. In Proc. of NSDI, 2009.

[47] B. Viswanath, A. Post, K. P. Gummadi, and A. Mislove. An analysis of social network-based sybil defenses. In Proc. of SIGCOMM, 2010.

[48] G. Wang, T. Konolige, C. Wilson, X. Wang, H. Zheng, and B. Y. Zhao. You are how you click: Clickstream analysis for sybil detection. In Proc. of USENIX Security, 2013.

[49] G. Wang, M. Mohanlal, C. Wilson, X. Wang, M. Metzger, H. Zheng, and B. Y. Zhao. Social turing tests: Crowdsourcing sybil detection. In Proc. of NDSS, 2013.

[50] G. Wang, B. Wang, T. Wang, A. Nika, H. Zheng, and B. Y.
Zhao. Whispers in the dark: Analysis of an anonymous social network. In Proc. of IMC, 2014.

[51] X. Wang, J. Zhu, A. Pande, A. Raghuramu, P. Mohapatra, T. Abdelzaher, and R. Ganti. STAMP: Ad hoc spatial-temporal provenance assurance for mobile users. In Proc. of ICNP, 2013.

[52] H. Yu, P. B. Gibbons, M. Kaminsky, and F. Xiao. Sybillimit: A near-optimal social network defense against sybil attacks. In Proc. of IEEE S\&P, 2008.

[53] H. Yu, M. Kaminsky, P. B. Gibbons, and A. Flaxman. Sybilguard: defending against sybil attacks via social networks. In Proc. of SIGCOMM, 2006.

[54] Z. Zhang, L. Zhou, X. Zhao, G. Wang, Y. Su, M. Metzger, H. Zheng, and B. Y. Zhao. On the validity of geosocial mobility traces. In Proc. of HotNets, 2013.

[55] Z. Zhu and G. Cao. Toward privacy preserving and collusion resistance in a location proof updating system. IEEE Transactions on Mobile Computing, 12(1):51-64, 2013. 